\title{
Maximizing Throughput of Cluster-Based WBAN with IEEE 802.15.6CSMA/CA
}

\author{
Pham Thanh Hiep \\ Le Quy Don Technical University, Ha Noi, Viet Nam \\ Yokohama National University, Yokohama, Japan \\ phamthanhhiep@gmail.com \\ Nguyen Huy Hoang \\ Le Quy Don Technical University, Ha Noi, Viet Nam \\ hoangnh@mta.edu.vn \\ Abstract
}

According to as urge in demand of wireless body area network, (WBAN), there are many researches on WBAN based on standard IEEE 802.15.6. These researches focused on physical layer, media access control (MAC) laygr and networklayer based on IEEE 802.15.6. Since network topology in the standard is defined asone hop star plus one, the previous research focused on one hop star topology and a multiple hopstechnic wasn't applied to WBAN. Based on network topology of WBAN, actuster-based meaning two hops technic is considered in this paper. Incluster-based WBAN, acluster header $(\mathrm{CH})$ forward the received data packet from a sensor to the coordinator therefore, an over concentration that is a reason of deterioration of performance, am be avoided and then the performance of system is improved. However, the optimal access probability that can achieve the highest through put is changed depending on a number of clusters. The optimization method of access probability is proposed and the calculation result shows that the cluster-based topology system out performs one hop syar topology;

Keywords: Optimizing access probability, Cluster-based wireless body area network, Maximizing throughput, CSMA/CA based on IEEE802.15.6

\section{Introduction}

Nowadays, many countries face elderly population (persons 65 years old and over), as number of senior citizens increasing all over the world. In order to survey health situation of elderly peoples under the limited financial resources and current medical service, the remote monitoring of body status and the surrounding environment is becoming more important. Furthermore, since many body functions are traditionally monitored and separated by a considerable period of time, it is hard for doctor to know what is really happening. This is reason why the monitoring of move ment and all body function in daily life are essential. One of the monitoring system is wireless body area network (WBAN). AWBAN consists of interconnected sensors, either placed around body or small enough to be placed inside the body. These sensors continuously monitor data and send it to coordinator; Coordinator gathers data of all sensor and sends to Healthcare center through existing network.

AWBAN system can be divided into 2 schemes; scheme 1: all sensors transmit the signal directly to the coordinator, scheme 2 : the sensor transmits the signal to the coordinator via multiple hops [1-5]. Inscheme 1, the sensor should use high power to transmit the signal 
because the coordinator isn't always close to. Therefore, the life time of sensors becomes shorter and each sensor causes the interference to other sensors initsarea. Moreover, the connection between sensors and the coordinator may be fails due to the interruption of the body, especially when the human is moving. Whereas, inscheme 2 , since each sensor transmits the signal to the neighbor sensor, the transmit power, the transmit area and the effective area are small. Therefore, the number of interfered sensors decreases and the lifetime of sensors increases. In additional, even the direct connection between the sensor to the coordinator is failed, the sensor can transmits to the coordinator via other sensors that connects to the coordinator. Therefore, in this paper, the multiple hops WBAN system is considered to analyze.

The multiple hops system is being researched in many literatures of many fields, e.g.adhoc network, mobile network, ITS system and soon [6-11]. However, in these systerns, sender(s) send the signal to receiver(s) via relay(s) and the relay just forwards the signal. In WBAN systems, each sensor forwards the signal while monitoring the situation of body and generating the vital signal by itself. In addition, the CSMA/CA based on standard IEEE 802.15.6 is assumed [12-18]. According to the definition of network topology in standard, two hops topology meaning cluster-based is cons dered. Member sensors transmit he data packet to their $\mathrm{CH}$ and $\mathrm{CHs}$ forward the received data packets to the coordinator. Since the cluster-based wireless sensors network is known as an, energy-efficiency topology and be researched in many literatures, in this paper, we focus on only the through put of system. An access probability of all sensors is optimized to obtain the maxim althrough put and the through put of cluster-based WBAN is compared to that of one hop star topology.

The rest of paper is organized as follows. We introduce the concept of multiple hops WBAN system and analyze the through put of 4 hop star WBAN in Section 2. Section 3 shows the system model and the through put of cluster-based CSMA/CAWBAN system. Finally, Section 4 concludes the paper.

\section{One-hop Star Topology for BAN}

\subsection{System Model}

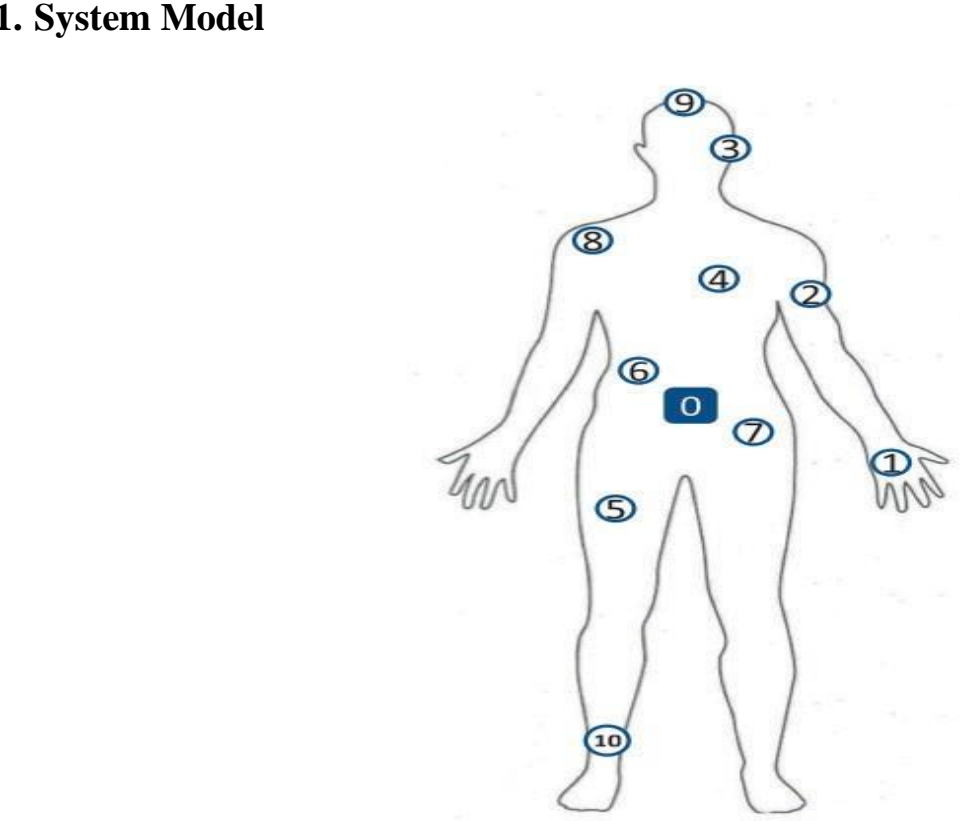

Figure 1. Body Area Network 
Figure 1 shows an example for WBAN system. There are a lot of sensors that distributed around the body to monitor the health situation. All sensors transmit the vital data toward the coordinator. In BAN system based on one-hop star topology, all sensors transmit directly its data to the coordinator. The vital data packet is generated at each sensor by its access probability. The carrier sense multiple access with collision avoidance (CSMA/CA) based on IEEE 802.15.6 is applied. After receiving the information data from the coordinator, the sensor can estimates a distance, a channel condition between the coordinator and itself. Thus the sensor adjusts its transmit power suitably to transmit the signal to the coordinator. Suitable transmit power means the signal to noise ratio (SNR) at receivers it equal to the threshold of desired SNR (dSNRthres).

\section{2 . CSMA/CA based on IEEE 802.15.6}

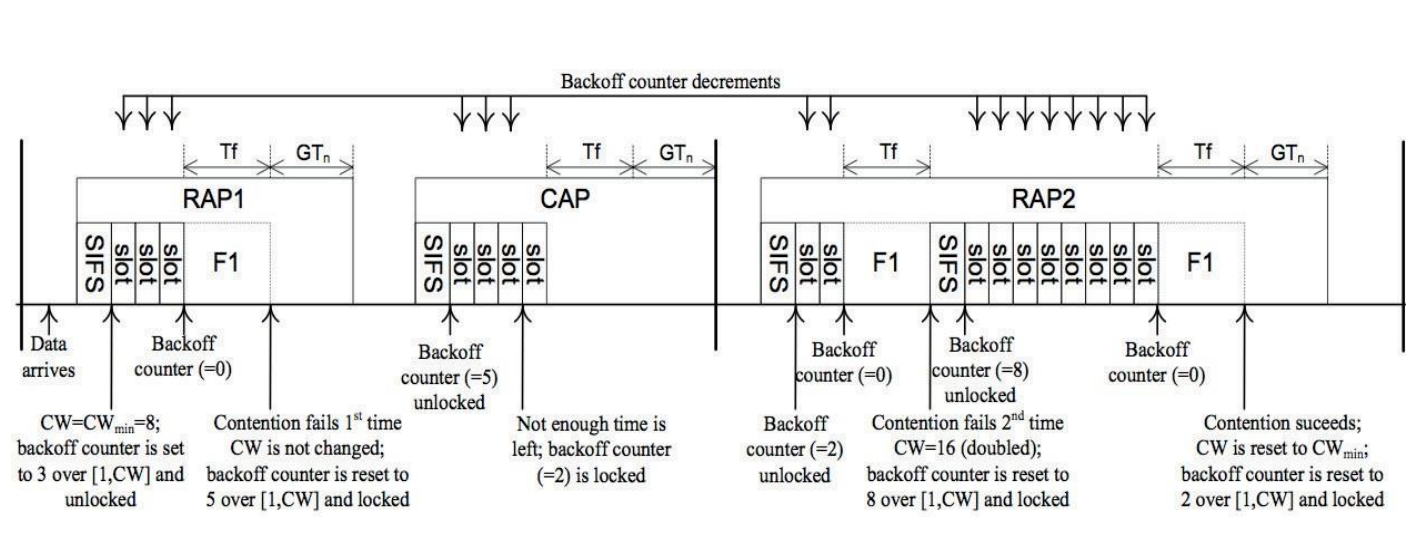

Figure 2. An Example of IEEE 802.15.6CSMA/CA Procedure

The outline of CSMAYCA based on IEEE 802.15.6 is described as follows, further details about the GSMA/CA procedure can be found in the standard [10]. As shown in Figure 2, in ESMA CCA, all sensors set its backoff counter to a random integer number uniformly distributed over the interval $[1, \mathrm{CW}]$ where $\mathrm{CW}$ is within $\left(C W_{\min }, C W_{\max }\right)$. The values of $C W \min$ and $C W \max$ vary depending on the user priorities as given in Table 1. A sensor starts decrementing the backoff counter by one for each idle CSMA slot of duration equal to $p$ CSMASlotLength. Once the backoffcounter reaches zero, the sensor transmits the frame. If the channel is busy because of a frame transmission, the sensor locks its backoff counter until the channel is idle. A sensor senses that the channel is busy if the SNR of received signal is higher than the threshold of effective SNR (eSNRthres). Normally, $d S N R$ thres $>e S N R$ thres. The transmission is failed if the sensor fails to receive an acknowledgement or group acknowledgement, however, in this paper, the sensor is assumed to transmit frame one by one. The $\mathrm{CW}$ is doubled for even number of failures. The $\mathrm{CW}$ is doubled until it reaches $C W \max$. Once the data transmission is successful, the $\mathrm{CW}$ is set to $C W_{\min }$.

The maximum through put is defined as the maximum number of MAC Layer Service Data Units (MSDUs) that can be transmitted in a unit time. Each MSDU carries additional overhead at MAC and PHY layers such as PHY preamble and PHY/MAC headers, control frames, inter frame spacing, and the backoff time. This additional over head affects the Maximum Through put of the network. Since all sensors can control its transmit power suitably to transmit the signal to the coordinator, the bit error rate (BER) is assumed to be considerably small and can be negligible. We assume that there are no packet losses due to buffer overflow. 


\section{Table 1. Contention Windown Bounds for CSMA/CA}

\begin{tabular}{ccc}
\hline Userpriority & $C \boldsymbol{W}_{\min }$ & $\boldsymbol{C W}$ max \\
0 & 16 & 64 \\
1 & 16 & 32 \\
2 & 8 & 32 \\
3 & 8 & 16 \\
4 & 4 & 16 \\
5 & 4 & 8 \\
6 & 2 & 8 \\
7 & 1 & 4 \\
\hline
\end{tabular}

The service time $(T)$ is defined as total time to transmit a packet included the backoff time $\left(T_{C W}\right)$, the time to transmit a data packet $\left(T_{D A T A}\right)$, interframes pacing $\left(T_{p S I F S}\right)$, the time of acknowledgement packet $\left(T_{A C K}\right)$ and delay time $(\alpha)$.

$$
T=T_{C W}+T_{D A T A}+T_{A C K}+2 T_{p S I F S}+2 \alpha \text {. }
$$

$T_{S}$ denotes a CSMA slot length. Hence, .

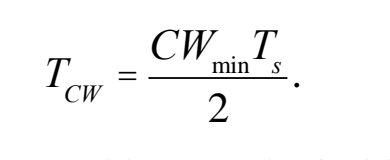

Since a data packet consists a preamble ( $\left(T^{\mathcal{B}}\right)$, physical header $\left(T_{P H Y}\right)$, MAC header $\left(T_{M A C}\right)$, MAC frame body $\left(T_{B O D Y}\right)$ and frame check sequence $\left(T_{F C S}\right)$, the time to transmit a data packet is represented as follows.

$$
T_{\text {DATA }}=T_{P}+T_{P H Y}+T_{M A C}+T_{B O D Y}+T_{F C S .}
$$

Since an immediate acknowledgement carries no payload, its transmission time is given by

\section{3 . Analysis Performance}

$$
T_{A C K}=T_{P}+T_{P H Y}+T_{M A C}+T_{F C S}
$$

The number of sensor on the body is denoted by $N$, and each sensor randomly and independently accesses as lot time with probability $\tau$. In this assumption, the probability $P$ idle that no sensor accesses a given slot is readily given by

$$
P_{\text {idle }}=(1-\tau)^{N} \text {. }
$$

Similarly, the probability $P$ suc that just one sensor accesses a given slot is expressed as

$$
P_{\text {suc }}=N \tau(1-\tau)^{N-1} \text {. }
$$

The collision time $T_{C}$ is defined as the duration of a period in which other stations cannot access the channel because a collision is occurring. However, as mentioned above, the sensor transmits the signal to the coordinator and waits for ACK packet from the coordinator. In case of collision, there is no ACK packet replied to the sensor, and then the sensor starts count down sits backoff time. It means the service time $T$ and the collision time $T_{c}$ are almost the same. In this paper, we assume $T=T_{c}$. Since a system slot-time $T_{s}$ elapses during an idle slot, we can derive the average slot duration 
as follows.

$$
E[\text { slot }]=P_{\text {idle }} T_{s}+P_{\text {suc }} T+\left(1-P_{\text {suc }}-P_{\text {idle }}\right) T_{c} .
$$

Finally, the system through put Thro as the average amount of information transmitted in to a slot. Note that $\mathrm{E}[\mathrm{P}]$ is the average MAC frame body size.

$$
\begin{aligned}
\text { Thro }_{s} & =\frac{P_{\text {suc }} E[P]}{E[\text { slot }]}, \\
& =\frac{P_{\text {suc }} E[P]}{P_{\text {idle }} T_{s}+P_{\text {suc }} T+\left(1-P_{\text {suc }}-P_{\text {idle }}\right) T_{c}} .
\end{aligned}
$$

The through put above is maximized as long as we minimize the term:

$$
f=\frac{P_{\text {idle }}\left(T_{s}-T\right)+T}{P_{\text {suc }}}
$$

The optimal value $\tau_{\text {opti }}$ that maximizes the system throughput is giyen by the solution of the equality $\frac{\partial f}{\partial \tau}=0$

$$
\left(1-\tau_{o p t i}\right)^{N}-\frac{T}{T}\left(10 \tau_{0 p t i}-\left(1-\left(1-\tau_{o p t i}\right)^{N}\right)\right)=0
$$

Under the condition $\tau<<1$, the approximation

$$
\left(1 \rightarrow-\tau_{\text {op }}\right) \approx 1-\hat{N} \tau_{g p t i}+\frac{N(N-1)}{2} \tau_{o p t i}^{2},
$$

holds, and hence the optimal yaluet opti can be found

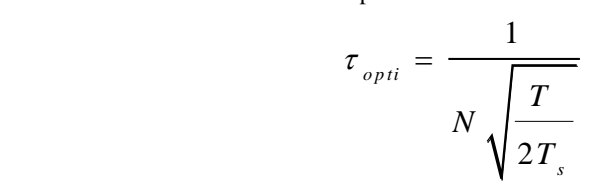

\subsection{Numerical Evaluation for One-Hop System}

In order to evaluate the theoretical analysis, the parameter that is summarized in Table 2, is used as anexample.

Figure 3 shows the throughput of system when the $\tau$ is changed. The number of sensors is changed, i.e., 5, 10, 15, 20. For each number of sensors, there are the optimal $\tau$ that reaches the maximum of throughput. The higher the number of sensors is, the lower the optimal $\tau$ is. It can be also recognized from Figure 4, the optimal au is calculated by (12) when the number of sensors increases. It means each sensor can transmit its packet with high probability when the number of sensors is small. On the other hand, as shown in Figures 3 and 4, when the number of sensors changes, the optimal $\tau$ is changed, however the maximum throughputs are almost the same. Even the sum of $\tau$ o fall sensors is fixed, the throughput decreases when the number of sensors increases. It means that even the access probability of each sensor is fixed, cluster-based topology, a sensor transmits the signal to cluster header instead of the coordinator, hence the number of sensors that accesses to the coordinator decreases and the throughput of system is expected to increase. Moreover, in one-hop star topology, to transmit 
directly to the coordinator, the transmit power of all sensor is high, especially the sensor is far from the coordinator. Additionally, some direct links from sensors to the coordinator maybe interrupted due to disturbance of body. Hence, the cluster-based topology should be considered.

\section{Table 2.}
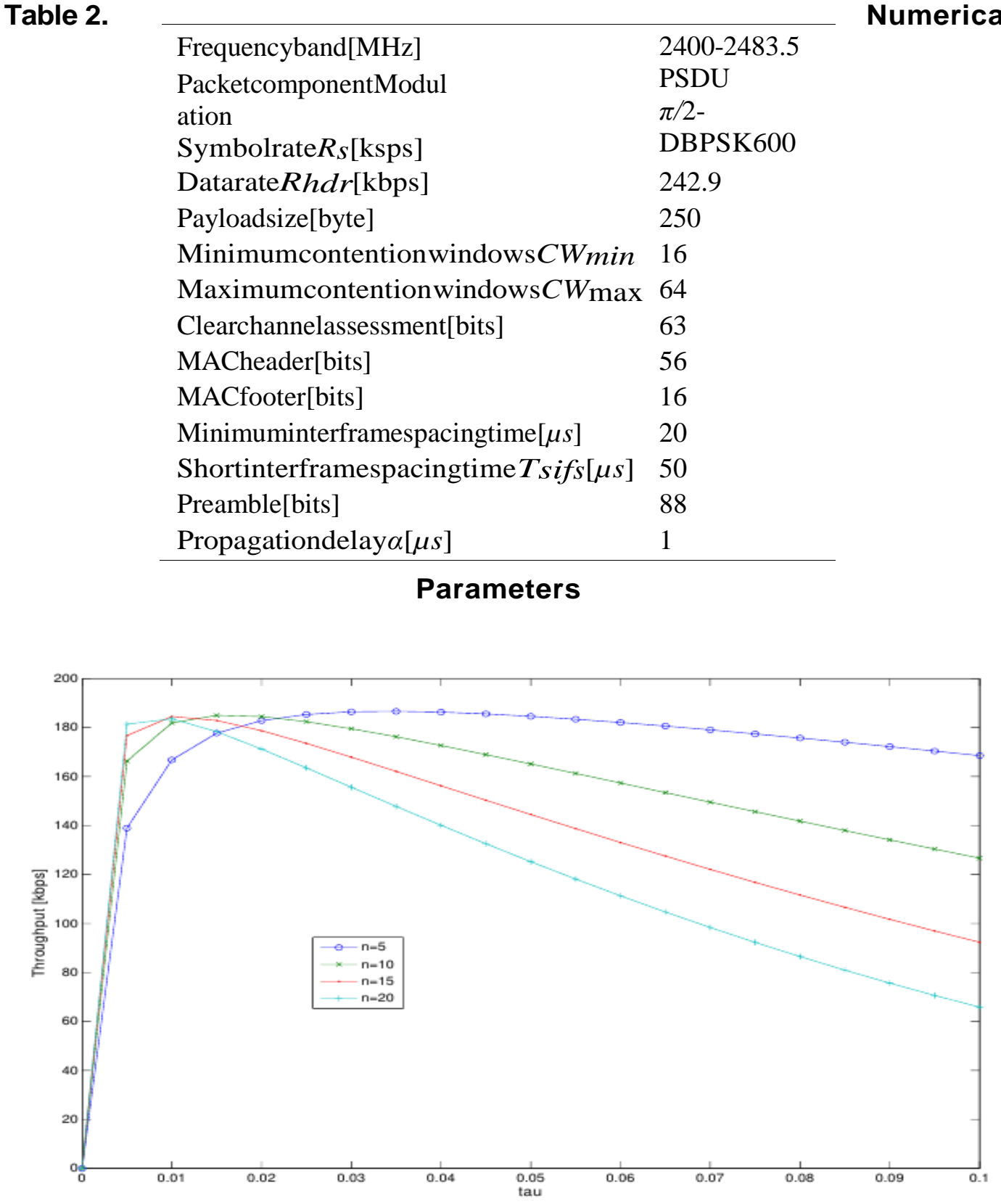

Figure 3. The Throughput of Some Numbers of Sensors, i.e., 5, 10, 15, 20 


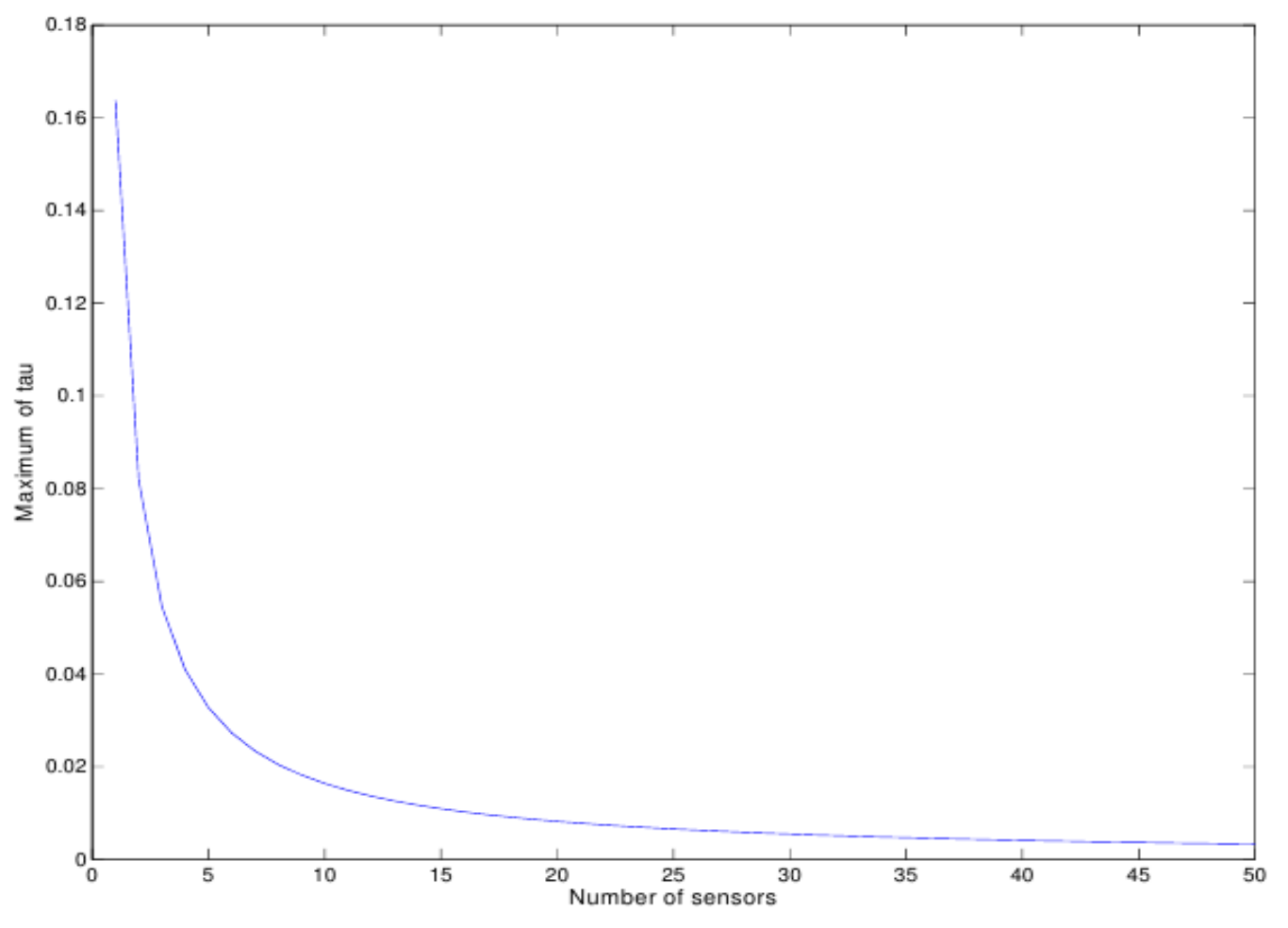

Figure $4 \tau_{\mathrm{opti}}$ Por each Number of Sensors

\section{Cluster-Based Topology System}

\subsection{System Model of Cluster-based Topology}

The system model of cluster-based topology is the same as the system model of one-hop star topology that is described in Section 2.1. However, in cluster-based topology, a sensor can transmit the signal to its cluster header $(\mathrm{CH})$ instead of the coordinator (Figure 5). Since all sensors generate a packet of vital data and transmit forward the coordinator via the $\mathrm{CH}$, the transmit power of all sensors and the throughput is expected to be improved. We assume that the transmission in a cluster is not effective to sensors in other clusters. After receiving the packet from a sensor, the $\mathrm{CH}$ immediately forwards the signal based on CSMA/CA algorithm of IEEE 802 15.6. The access probability $T$ of all sensors in cluster-based topology is assumed to be the same as the access probability of one-hop topology. 


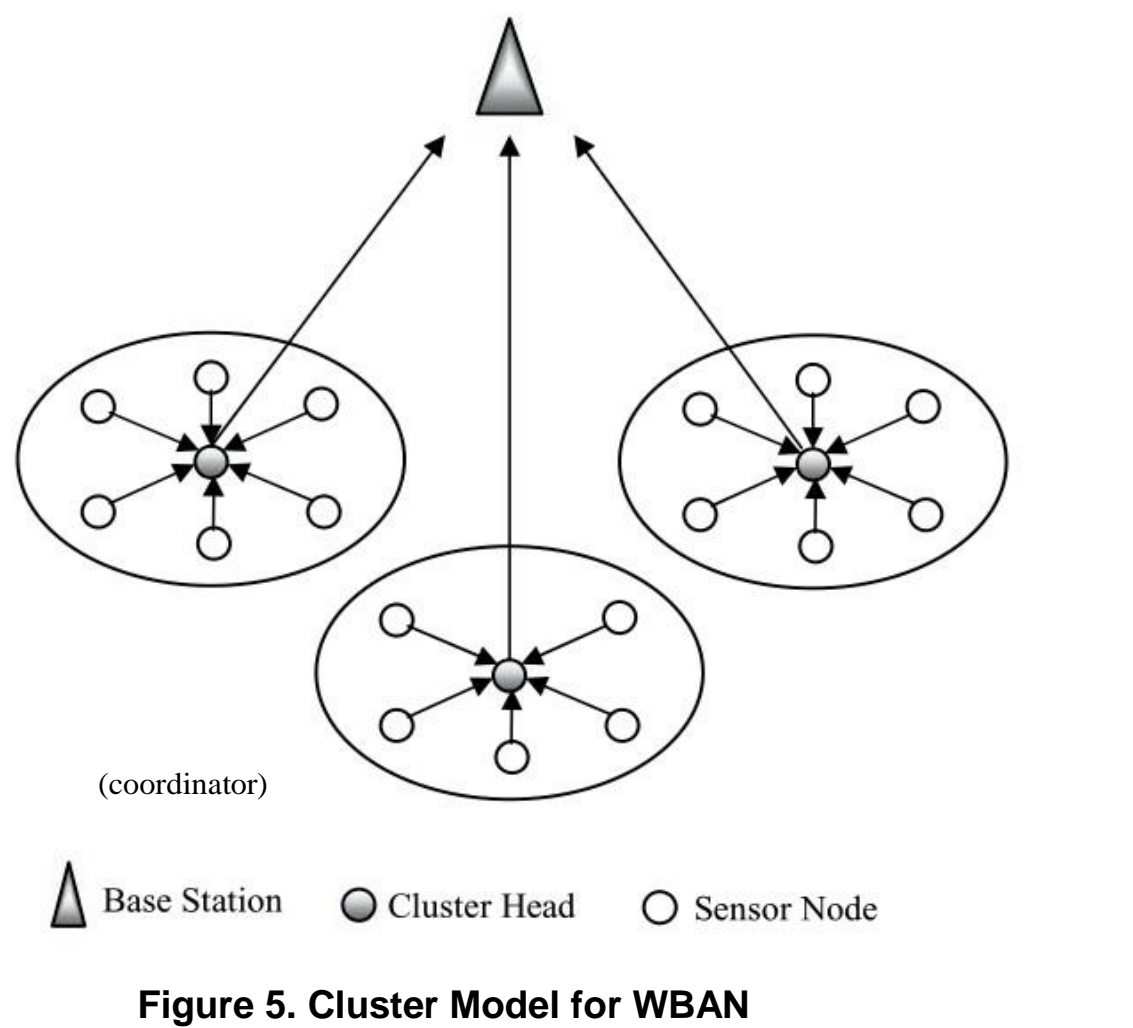

Figure 5. Cluster Model for WBAN

\subsection{Optimizing access probability for WBAN}

The number of sensors in each cluster (Not includes the $\mathrm{CH}$ ) and the number of cluster are denoted by $N_{s}$ and $N$, respectively. Thys

$$
N_{s}=\frac{N}{N_{c}}-1
$$

Each cluster can be considered as a none-hop star topology that was explained above. Therefore, the access probability of $\mathrm{CH}$ is described as follows.

$$
\tau_{c}=P_{s u c}^{s} \tau+\tau,
$$

here $p_{s y c}=N_{s} \tau(1-\tau)^{N_{s}-1}$ denotes the successful probability of all sensors in one cluster.

All CHs and the coordinator constructs an one-hop star topology, therefore, the optimal access probability for each $\mathrm{CH}$ can be calculated as (12). From (12) and (14), we have

$$
\frac{1}{N_{c} \sqrt{\frac{T}{2 T_{s}}}}=P_{s u c}^{s} \tau+\tau,
$$

therefore, the optimal access probability of all sensors in cluster-based topology is represented as 


$$
\tau_{\text {opti }}^{c}=\frac{\sqrt{N_{c}^{2}+4 N\left(N-N_{c}\right) \tau_{\text {opti }}}-N_{c}}{2\left(N-N_{c}\right)}
$$

Notice that $\tau_{\text {opti }}$ is the optimal access probability of sensor in one-hop star topology in (12).

\subsection{Numerical Evaluation for Cluster-Based Topology}

The system model of cluster-based topology is the same as the original WBAN system (Table 2). The number of all sensors is 50 and the number of clusters is changed. Figures 6 and 7 show comparing the optimal access

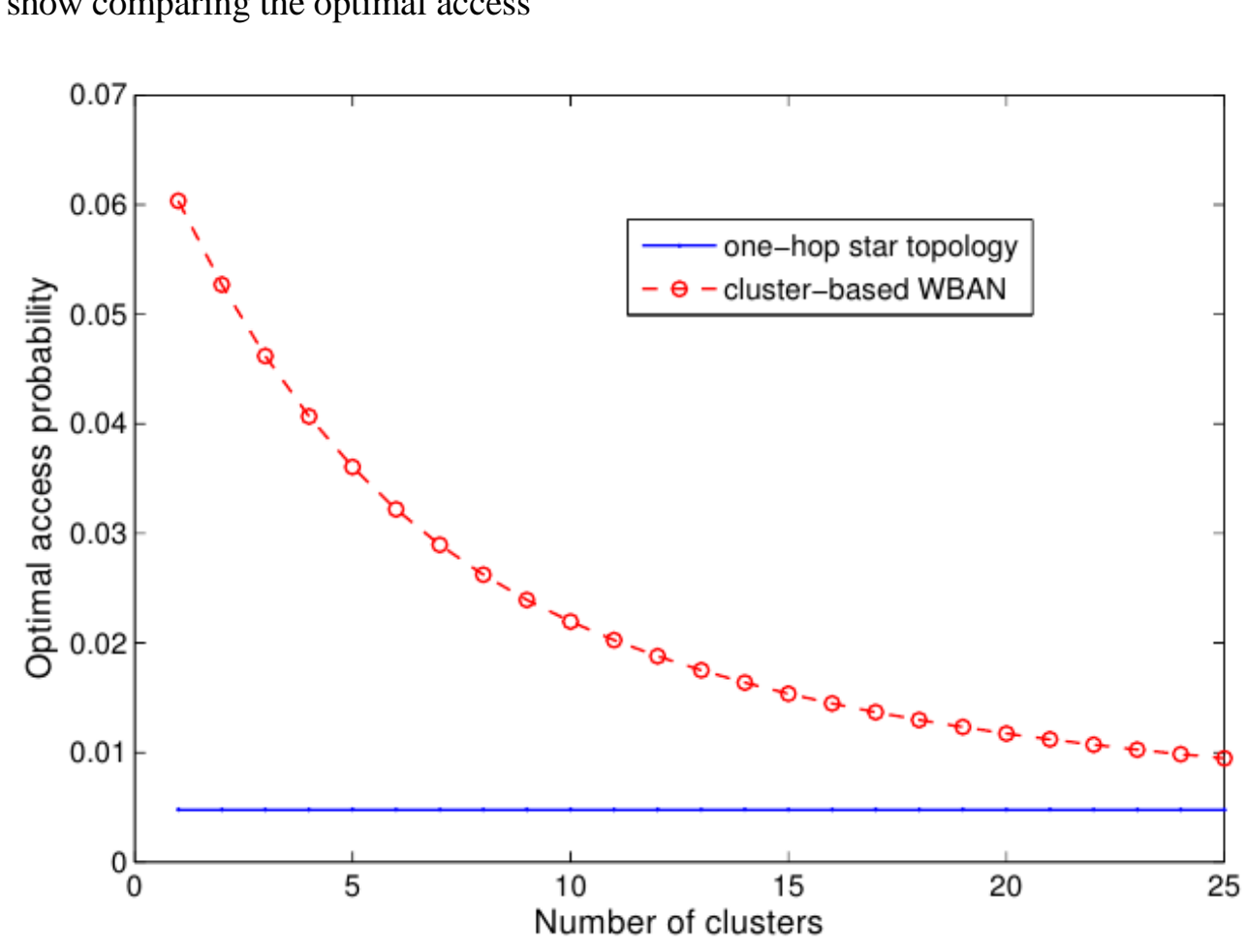

Figure 6. The Optimal Access Probability

Probability and the throughput of one-hop star topology to cluster-based topology, respectively. As shown in Figure 6, the optimal access probability of cluster-based topology is higher than that of one-hop star topology. The sensors in cluster-based can transmit with higher access probability because they transmit to their $\mathrm{CH}$, therefore the collision is small.

With the higher access probability, the throughput of cluster-based topology is also higher than that of one-hop star topology, especially when the number of clusters is small (Figure 7). 


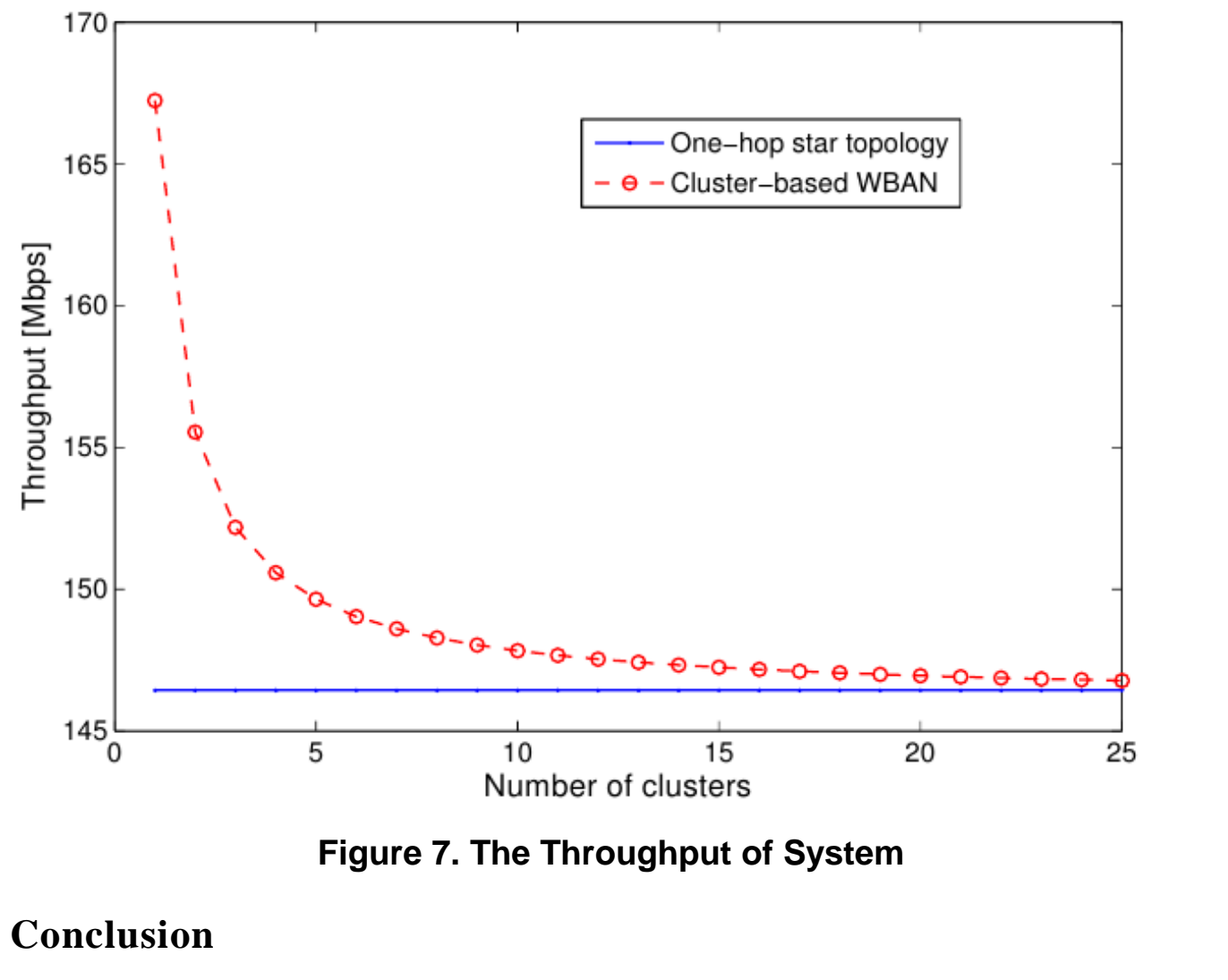

In this paper, the WBAN with CSMA/CA protocol based on standard IEEE 802.15.6 was analyzed. The accessuprobability of all sensors was optimized to obtain the highest throughput based on both one hop star topology and cluster-based topology. As proposed system model, the throughpuf of cluster-based topology is higher than that of one hop star topology. However, in the data packet that transmitted to the coordinator, the data packets of CHs is estimated to occupy the high percent, especially when the number of clusters is low. The fairness of transmission data for all sensors is left to the future work.

\section{References}

[1] G. Z. Yang, "Body sensor networks (chapter: Wireless communication)", Springer, (2006).

[2] E. Jovanov, A. Milenkovic, C. Otto and P. A. Degroen, "Wireless body area network for intelligent motion sensors for computer assisted physical rehabilitation", JNER2, no. 6, (2005), pp. 16-23.

[3] S. Marinkovi, E. M. Popovici, C. Spagnol, S. Faul and W. P. Marnane, "Energy-efficient low duty cycle MAC protocol for wireless body area networks", IEEE Trans. Inf. Tech-nol. Biomed, vol. 13, no. 6, (2009), pp.915-925.

[4] G.-Z. Yang, "Body Sensor Networks", Springer, London, (2006), pp. 125-127.

[5] W. Ye and H. J. Estrin, "An energy-efficient MAC protocol for wireless sensor networks", In Proc. Of Twenty-First Annual Joint Conference of the IEEE Computer and Communications Societies, Miami, USA, (2002), pp.1567-1576.

[6] E.-S. Jung and N. H. Vaidya, "A Power Control MAC Protocol for AdHoc Networks", MOBICOM02, (2002) September 23-28, Atlanta, Georgia, USA.

[7] N. F. Timmons and W. G. Scanlon, "Analysis of the performance of IEEE 802.15.4 for medical sensor body area networking", in the Proc. Of First Annual IEEE Communications Society Conference on Sensor and AdHoc Communications and Networks (IEEESECON2004), (2004), pp.16-24.

[8] "Wireless LAN Medium Access Control (MAC) and Physical Layer (PHY) specifications: Medium access control quality of services” IEEE 802. 11e Std Amend mentto Part11, enhancements, (2005). 
[9] K. Akkaya and M. Younis, "A survey on routing protocols for wireless sensor networks," AdHoc Networks vol. 3, Issue 3, (2005), pp. 325-349.

[10] "Wireless Medium Access Control (MAC) and Physical Layer (PHY) Specifications for Wireless Personal Area Networks (WPANs) use dinor around a body", IEEEP 802.15.6/D01, (2010).

[11] G. Bianchi, "Performance analysis of the IEEE 802.11 distributed coordination function", IEEE Journal on Selected Areas in Communications, vol. 18, Issue 3, (2000) March.

[12] K. S. Kwak, S. Ullah and N. Ullah, "An Overview of IEEE 802.15.6 Standard", $3{ }^{\text {rd }}$ International Symposium on Applied Sciences in Biomedical and Communication Technologies (ISABEL), (2010).

[13] F. Martelli, C. Buratti, R. Verdone, " On the performance of an IEEE 802.15.6 Wireless Body Area Network," European Wireless 2011, (2011) April 27-29, Vienna, Austria.

[14] S. Ullah, H. Higgins, B. Braem, B. Latre, C. Blondia, I. Moerman, S. Saleem, Z. Rahmanand K. S. Kwak, "A Comprehensive Survey of Wireless Body Area Networks: On PHY, MAC and Network Layer Solutions", Journal of Medical Systems, INPRESS, DOI:10.1007/s10916-010-9571-3.

[15] B. Zhen,H. B. Li and R. Kohno, "IEEE body area networks and medical implant communications", in the Proc. Of the ICST 3rd International Conference on Body Area Networks, Tempe, Ariz, USA, (2008).

[16] A. W. Astrin, H.-B. Li and R. Kohno, "standard ization for body area networks", IIEICE Transactions on Communications, vol. E92.B, no.2, (2009), pp.366-372.

[17] N. Bradai, S. Belhaj, L. Chaari and L. Kamoun, "Study of Medium Access Mechanisms ander IEEE 802.15.6 Standard", IFIPWMNC, (2011).

[18] S. Ullah, H. Higgins, B. Braem, B. Latre, C. Blondia, I. Moerman, S. Saleem, Z. Rahman and K. S. Kwak, "A Comprehensive Survey of Wireless Body Area Networks On PHY, MAC and Network Layers Solutions", MedSyst, Springer Science Business Media, LLC2010, vol. 36, (2012), pp.1065-1094.

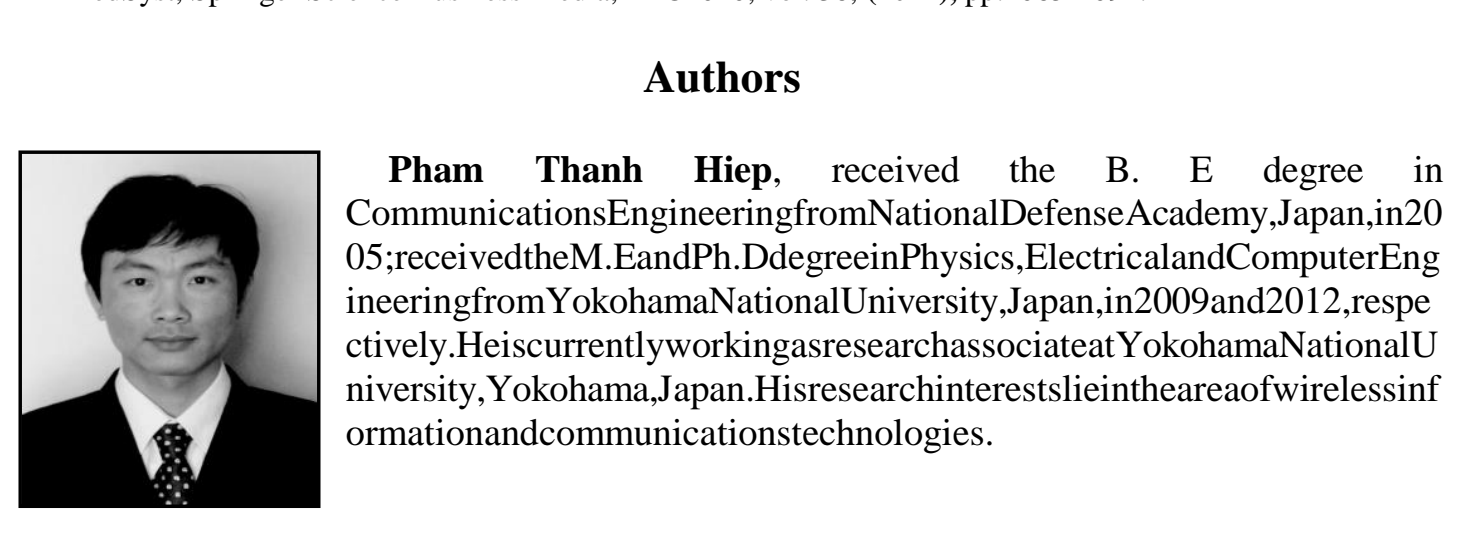

\section{8}

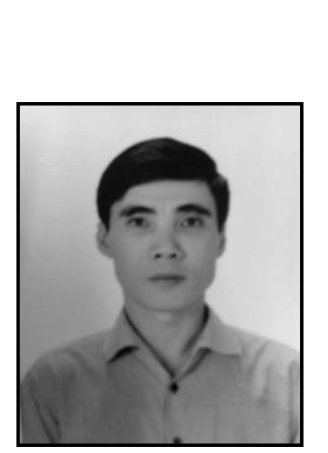

Nguyen Huy Hoang, received B.E, M.E and $\mathrm{PhD}$ degree in Electronic Engineering from Le Quy Don Technical University, Vietnam, in 1986, 1997 and 2007, respectively. He is currently lecturer at Le Quy Don Technical University, Vietnam. His research concetrates on the area of wireless information, communication technologies, microwave electronic circuit. 
International Journal of Multimedia and Ubiquitous Engineering Vol.9, No.5 (2014)

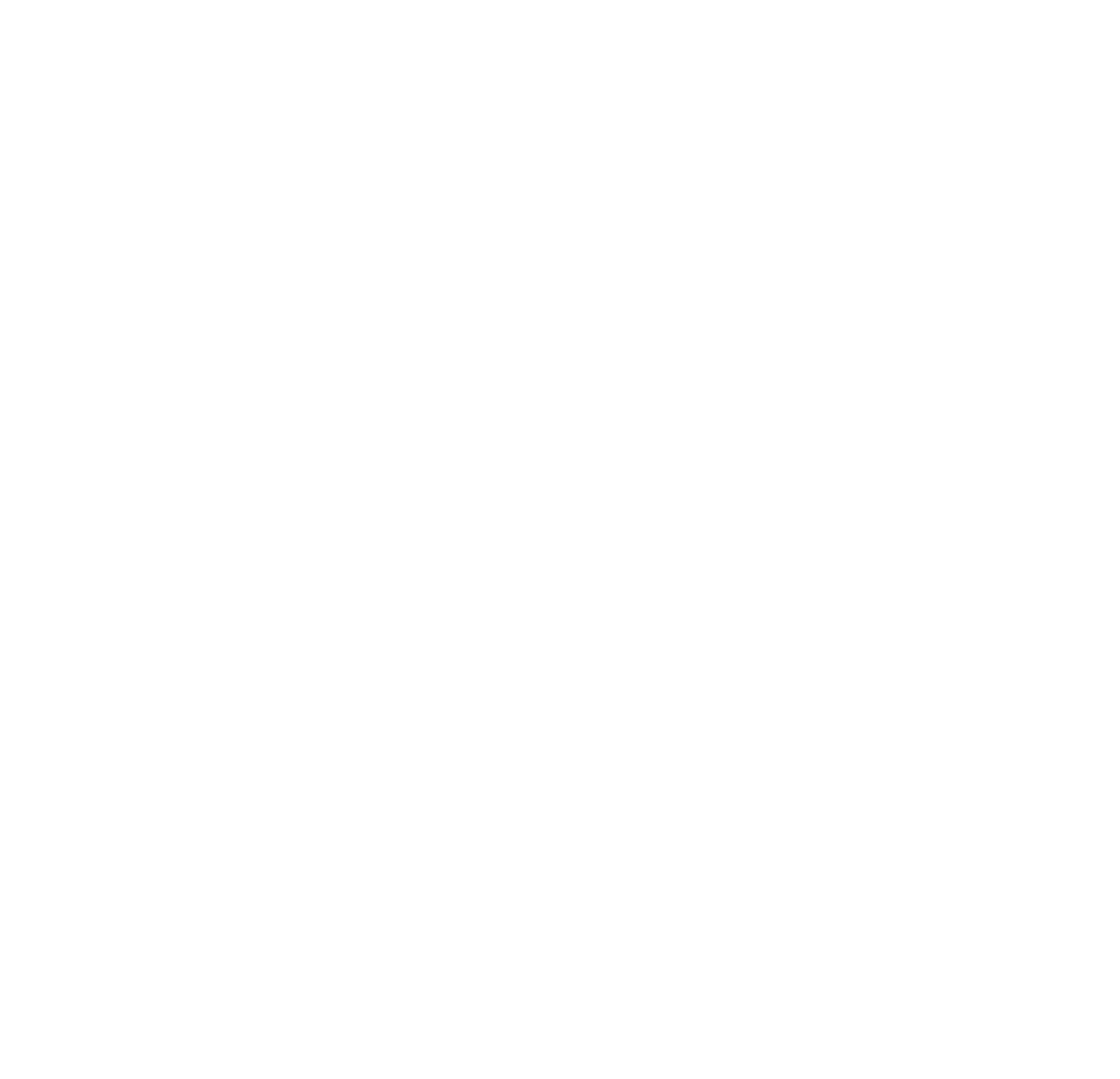

